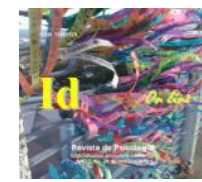

\title{
Aleitamento Materno Exclusivo até os seis meses de vida na Região do Araripe
}

\author{
Cícero Cruz Macêdo ${ }^{1}$; Luiz Carlos de Abreu ${ }^{2 ;}$ Andréia Manoele Henrique ; Maria de Fátima Lopes da Cruz ; Gislene \\ Farias de Oliveira ${ }^{l}$; João Saraiva da Cruz Neto ${ }^{5}$; Leonardo Lopes da Cruz ${ }^{5}$; Maria Eliane Pierre Martins ${ }^{1}$; Sionara Melo \\ Figueiredo Carvalho ${ }^{l}$
}

Resumo: O aleitamento materno tem se constituído uma das medidas mais importantes e necessária para um melhor desenvolvimento infantil nos primeiros meses de vida. A literatura tem demonstrado através de inúmeros estudos que esta prática é capaz de prevenir distúrbios nutricionais e metabólicos, inclusive em idades mais avançadas. Desde maio de 2009, o Hospital Municipal Marcelino da Silva Mudo (HMMSM), em Ipubi - PE, passou a oferecer como estratégia de saúde, um programa de aleitamento materno exclusivo. O Método constou do acompanhamento de crianças em aleitamento materno exclusivo até os seis meses de vida, no HMMSM, através de orientação e apoio de equipe multidisciplinar, evitando a prática do aleitamento misto ou artificial. Dentre os principais objetivos do presente estudo estão: a) Funcionar como iniciativa de saúde destinada a realizar assistência continua através de equipes multiprofissionais; b) Desenvolver ações de promoção, prevenção e apoio, realizando diagnóstico precoce, tratamento e reabilitação, características do nível primário de atenção; c) Trabalhar no seu território de abrangência - a área que está sob sua responsabilidade. Resultados: Entre 2009 a 2012, o número de consultas em crianças menores de 6 meses cresceu de 357 paara 835 e, o aleitamento materno exclusivo cresceu de $74,63 \%$ para $78,30 \%$ de 2008 a 2012. Segundo a SIAB a taxa de arreia em menores de 1 ano, caiu de 5 em 2008 para nenhum caso em 2012. O número de hospitalizações por pneumonia, caíram de 7 casos em 2008 para nenhum caso em 2012. Conclusão: A implantação do Programa trouxe benefícios para as crianças, mães e para o próprio município, que reduziu em $67 \%$ (sessenta e sete por cento) os atendimentos e internamentos em crianças menores de 2 (dois) anos, conseqüentemente reduzindo os custos municipais com a saúde, nesta dimensão. Portanto, este trabalho reafirma que o estímulo ao aleitamento materno exclusivo, é uma fórmula relativamente econômica e segura de promoção da saúde, devendo ser incentivada como prioritária, enquanto política pública.

Palavras-Chave: Aleitamento materno; Saúde infantil; Promoção da saúde

\section{Exclusive breastfeeding until six months of life in the Araripe Region}

\begin{abstract}
Breastfeeding has been one of the most important and necessary as for better child development in the first months of life. The literature has shown through numerous studies that this practice is able to prevent metabolic and nutritional disorders, including at older ages . Since May 2009, the Municipal Hospital Marcelino da Silva Mute ( HMMSM ) in Ipubi - PE, began offering as an health strategy , a program of exclusive breastfeeding. The method consisted of monitoring of children exclusively breastfed until six months of life, in HMMSM through guidance and support of a multidisciplinary team , avoiding the practice of mixed or artificial feeding. Among the main objectives of this study are : a) Serve as health initiative designed to conduct assistance continues through multidisciplinary teams, b ) Develop health promotion, prevention and support,
\end{abstract}

\footnotetext{
${ }^{1}$ Professor da Universidade Federal do Ceará - UFC;

${ }^{2}$ Professor da Faculdade de Medicina do ABC, Santo André, São Paulo;

${ }^{3}$ Enfermeira. Especialista em Obstetrícia.

${ }^{4}$ Médica Especialista em Saúde Pública e Saúde da Família;

${ }^{5}$ Médico. Estado do Ceará.

Correspondente: E-mail: cíceropediatra@hotmail.com
} 
making early diagnosis, treatment and rehabilitation, the primary characteristics of attention, c ) Work on your coverage area - the area that is under your responsibility . Results: Between 2009-2012, the number of consultations in children under 6 months has grown from 357 to 835, exclusive breastfeedinghas grown from $74.63 \%$ to $78.30 \%$ from 2008 to 2012 . According to the SIAB the gear ratio among children under 1 year fell from 5 in 2008 to no cases in 2012. The number of hospitalizations for pneumonia fell from 7 cases in 2008 to no cases in 2012. Conclusion : The implementation of the program has brought benefits for children , mothers and the municipality itself, which reduced by $67 \%$ (sixty -seven percent ) attendances and hospitalizations in children under two (2 ) years, thus reducing municipal costs with health, this dimension . Therefore, this study reaffirms that the stimulus to exclusive breastfeeding, is a relatively safe and economical formula for health promotion, and it should be encouraged as a priority, while public policy.

Keywords : Breastfeeding, Child Health, Health Promotion

\section{Introdução}

O aleitamento materno tem se constituído uma das medidas mais importantes e necessária para um melhor desenvolvimento infantil nos primeiros meses de vida. A literatura tem demonstrado através de inúmeros estudos que esta prática é capaz de prevenir distúrbios nutricionais e metabólicos, inclusive em idades mais avançadas.

Mesmo com toda propaganda de incentivo governamental ao aleitamento materno, desde a década de 80, no Brasil ainda tem se registrado baixos índices de amamentação exclusiva de bebês até sexto mês de vida. Pesquisa do Ministério da Saúde tem reportado aumento nos índices de aleitamento exclusivo até o quarto mês de vida, em torno de 35,5\% em 1999 para 51,2\% em 2008. Porém, ao sexto mês, praticamente não foi alterado. (MINISTÉRIO DA SAÚDE, 2009)

Incentivar ao aleitamento materno tem sido prática comum entre profissionais de saúde no Brasil, que relatam índices relativamente satisfatórios de adesão das mães a essa modalidade. Pesquisas apontam que entre $35 \%$ a 38,6\% dos bebês são mantidos em aleitamento exclusivo até o sexto mês (OLIVEIRA, CAMACHO, TEDSTONE, 2003; NARCHI, FERNANDES e GOMES, 2005 ; FALEIROS et al, 2005). Carrascoza (2007) relatou índice de 47,5\% de crianças em grupo que fora incentivado, em aleitamento materno exclusivo até o sexto mês. O autor reportou que tal índice se deveu às atuações efetivas da equipe interdisciplinar, a qual acompanhava as mães e os bebês, nos encontros por ocasião das consultas pós natais, com esclarecimento de dúvidas, discussão sobre a prática da amamentação e tratamentos de dificuldades diversas com o aleitamento.

Esses índices, ainda abaixo do preconizado pela Organização Mundial da Saúde (OMS), que defende que $100 \%$ das crianças deveriam se amamentadas exclusivamente até o sexto mês (OLIVEIRA, CAMACHO, TEDSTONE, 2003; NARCHI, FERNANDES e GOMES, 2005; FALEIROS et al, 2005; CARRASCOZA, 2007), mostram-se elevados em comparação a dados de 
http://idonline.emnuvens.com.br/id

ISSN on-line: 1981-1179

outros estudos sobre o índice de aleitamento em crianças no Brasil, cujas mães não fizeram parte de programas de incentivo ao aleitamento exclusivo e que assim, não receberam nenhum tipo de ajuda na manutenção desta prática (KUMMER, et AL, 2000; PASSOS, LAMOUNIER, SILVA, FREITAS e BAUDSON, 2000). Informações do Ministério da Saúde (2009) apontam uma média de 9,3\% de mulheres brasileiras que amamentam seus bebês de exclusiva até o sexto mês de vida.

Portanto pode-se inferir que as estratégias de incentivo e promoção ao aleitamento materno exclusivo são importantes e necessárias para a manutenção da exclusividade na amamentação e na prevenção do desmame de forma precoce. Dentre algumas dessas estratégias, há a Iniciativa Hospital Amigo da Criança, que teve início em 1990, através do Fundo das Nações Unidas para a Infância UNICEF, conjuntamente com a OMS. Dentre os objetivos dessa iniciativa, considera-se a mobilização dos funcionários de maternidades e hospitais, para que Sigam rigorosamente, as condutas e rotinas preconizadas como adequadas à prática do aleitamento. Dessa forma, obtém-se o título de Hospital Amigo da Criança, como forma de continuar motivando a instituição a cumprir os dez passos para o incentivo ao aleitamento. Dentre eles, o de encorajar a formação de equipes de apoio à amamentação, encaminhando as mães logo após a alta hospitalar (VANNUCHI, MONTEIRO, RÉA, ANDRADE e MATSUO, 2008).

Dos fatores de risco a manutenção do aleitamento exclusivo, pode-se destacar a utilização da chupeta (RAMOS, ALMEIDA, ALBERTO, TELES, SALDIVA, 2008), a primeira gestação, (FRANÇA, BRUNKEN, SILVA, ESCUDER, VENÂNCIO, 2007; ALVES, GOULARD, COLOSIMO, GOULARD, 2008) e o fato da mãe trabalhar fora do lar (OLIVEIRA, CAMACHO, TEDSTONE, 2003; MASCARENHAS, ALBERNAZ, SILVA e SILVEIRA, 2006). Outros estudos mostram que é crescente a participação das mulheres no mercado de trabalho e, que isso tem elevado o desmame precoce (SCHWARTZ, D’ARCY, GILLESPIE, BOBO, LONGEWAY e FOXMAN, 2002; DEARDEN et al, 2002). Os resultados sugerem que incentivar o aleitamento materno poderia conduzir a índices menores de desmame precoce, entre mães que trabalham fora do lar.

O objetivo do presente estudo foi discutir a efetividade do trabalho de incentivo ao aleitamento materno exclusivo no município de Ipubi, estado de Pernambuco, que desde maio de 2009, passou a oferecer como estratégia de saúde, um programa de aleitamento materno exclusivo, no Hospital Municipal Marcelino da Silva Mudo (HMMSM).

\section{Método}

O estudo se desenvolveu em Ipubi, município do sertão pernambucano que faz parte da Mesorregião do Araripe (Figura 1). Uma região que representa 18,8\% do território estadual e abrange

Id en line Revista de Psicologia. Ano 7, No. 21, Novembroo/2013 - ISSN 1981-1179. Edição eletrônica em http://idonline.emnuvens.com.br/id 
ainda, os municípios de Araripina, Cedro, Bodocó, Exu, Moreilândia, Granito, Serrita, Santa Filomena, Ouricuri, Verdejante, Santa Cruz, Trindade e Parnamirim.

Já teve como atividade predominante a agricultura, hoje tem a exploração da gipsita como a maior atividade econômica. Esta região concentra $40 \%$ das reservas de gipsita, sendo um dos maiores produtores de gesso e derivados, detendo $95 \%$ da produção nacional. Esta realidade gera cerca de doze mil empregos diretos, sendo 60 mil indiretos (IBGE, 2008).

Mesmo assim ainda são produzidas hortaliças, grãos, mandioca, além da criação de bovinos e aves, que junto com a apicultura são outras fontes de renda no município.

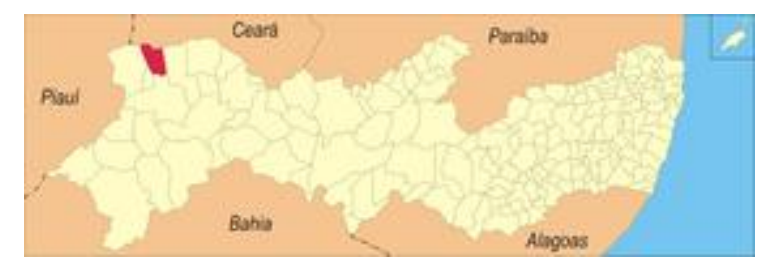

Figura 1 - Localização de Ipubi em Pernambuco Fonte: IBGE, 2008.

A estratégia do Programa constou basicamente do acompanhamento exclusivo de crianças em aleitamento materno exclusivo até os seis meses de vida, no Hospital Municipal Marcelino da Silva Mudo (HMMSM). Esta se deu através de orientação e apoio de equipe multidisciplinar, evitando-se a prática do aleitamento misto ou artificial. Dentre os principais objetivos do Programa foram: a) Funcionar como iniciativa de saúde destinada a realizar assistência continua através de equipes multiprofissionais; b) Desenvolver ações de promoção, prevenção e apoio, realizando diagnóstico precoce, tratamento e reabilitação, características do nível primário de atenção; c) Trabalhar no seu território de abrangência - a área que está sob sua responsabilidade.

O estudo, de natureza qualitativa e descritiva, foi feito através dos prontuários e registros do Hospital, sobre as mães participantes do programa de Aleitamento Materno. Buscou conhecer os resultados em termos dos principais indicadores nesta dimensão, a saber: adesão ao programa, medida pelo número de mães cadastradas no programa; número de consultas após o nascimento do bebê, as taxas de desnutrição, número de mães desistentes, número de mães em aleitamento exclusivo, dentre outros. Tais indicadores estão disponíveis nos arquivos do Hospital Municipal Marcelino da Silva Mudo (HMMSM), bem como disponibilizados no Sistema de Informação da Atenção Básica - SIAB, que é um instrumento gerencial utilizado para Sistemas Locais de Saúde. Foi implantado em 1998 com a função de monitorar e avaliar as ações da atenção básica, disponibilizando sua base de dados na internet, como ação estratégicas da política Ministerial da Saúde. Objetiva também fornecer informações para orientar tomada de decisão dos gestores do SUS, e de outras instâncias sociais.

Id en line Revista de Psicologia. Ano 7, No. 21, Novembroo/2013 - ISSN 1981-1179

Edição eletrônica em http://idonline.emnuvens.com.br/id 


\section{Resultados e Discussão}

Dados do HMMSM, apontaram que, entre 2009 a 2010, o número de consultas em crianças menores de seis meses de idade, cresceu de 357 em 2009 para 835 em 2012. Até setembro de 2013 esse número já era de 795 consultas realizadas (Gráfico 1).

Gráfico 1 - Número de consultas em crianças menores de 6 meses de idade. Ipubi-PE, 2009-2013

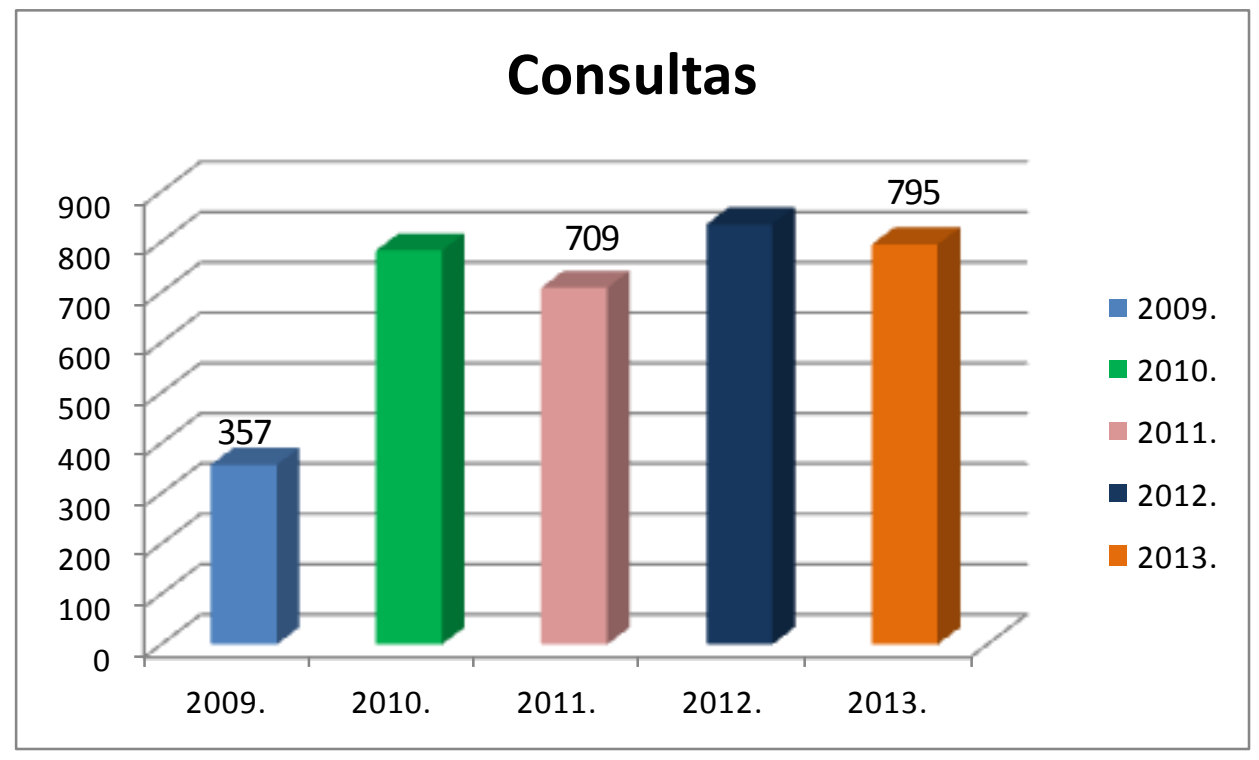

Fonte: HMMSM

Em termos de aleitamento materno exclusivo ao sexto mês de vida, esses números se revelam muito superiores ao de outros estudos, como por exemplo, no trabalho de Rea et al (1997), com mulheres trabalhadoras em São Paulo, onde no sexto mês de vida, $40 \%$ das crianças amamentavam ainda ao seio, mas nenhuma de forma exclusiva. Um estudo de Damião (2008) encontrou um percentual de $11,2 \%$ de mães que trabalhavam e que mantiveram o aleitamento materno, não de maneira exclusiva. Índices menores foram encontrados por Vianna, Rea e Venâncio e Escuder (2007), que relataram que apenas 5,3\% das mulheres trabalhadoras, participantes do estudo amamentavam aos quatro meses de vida dos bebês. Índices como esse, tendem a ser encontrados em mães que não participam de um Programa de Incentivo ao Aleitamento Materno. Isso ocorre geralmente por não receberem informações adequadas sobre aleitamento e sobre como superar as dificuldades no processo. 
As desistências na adesão ao programa caíram de 52 em 2009 para 22 em 2010. Esses números por si representam um ganho importante, pois pode-se inferir que é previsível que mães que estejam participando de um Programa de Incentivo ao Aleitamento Materno estejam mais motivadas e dispostas a amamentar. Contudo, deve-se levar em conta que, na atualidade, as mães tendem a trabalhar como forma de complementarem a renda familiar e, diante da legislação trabalhista, geralmente são oficializadas formalmente. Portanto, sabe-se que algum percentual de desistência pode se dever ao retorno ao trabalho, entre o terceiro e o quinto mês após o nascimento do bebê. Atualmente, o Ministério da Saúde tem enfrentado essa situação, remunerando com o benefício da licença-maternidade extendida até o sexto mês, quando a mãe se encontra amamentando (MINISTÉRIO DA SAÚDE, 2009).

Os números de aleitamento misto caíram. Antes era de 11 crianças do programa em 2009 e passou em 2010 para 4 crianças em aleitamento misto, antes dos seis meses de idade. O número de mães que mantiveram apenas o aleitamento artificial, antes dos seis meses se manteve constante: 3 em 2009 e 3 em 2010.

Borges e Philippi (2003) informam que há uma insegurança materna quando da aproximação do retorno ao trabalho. E que isso pode vir a ser um fator de risco na manutenção do aleitamento. Assim, participar em programas de incentivo, onde há profissionais para disponibilizar informações adequadas e práticas sobre aleitamento, pode representar um fator protetivo a mães e a criança, no sentido de melhorar os números da manutenção do aleitamento materno exclusivo até os seis meses de idade.

No mesmo período (2009-2010), o número de nascidos vivos, salta de 399 para 626, um incremento de $56,89 \%$. Caiu o número de bem como cai o número de hospitalizações por pneumonia em crianças menores de 4 anos de idade de 61 casos em 2009 para 34 casos em 2012, uma queda de $45,16 \%$ (Gráfico 2). 
Gráfico 2 - Número de hospitalizações por Pneumonia em crianças menores de 4 anos de idade. Ipubi-PE, 2008-2012.

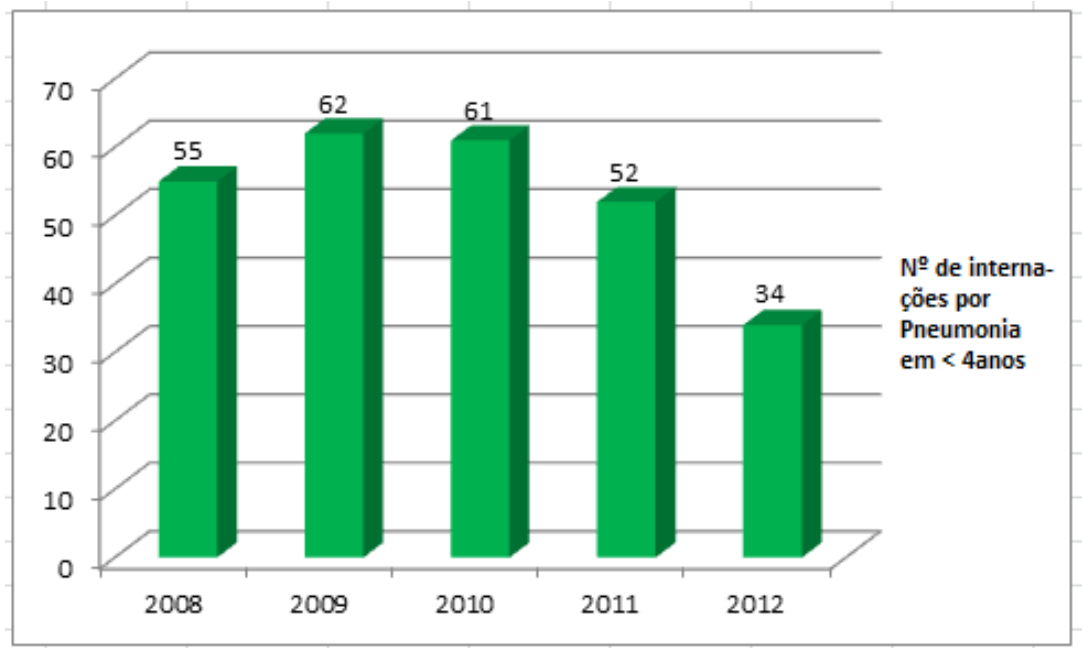

Fonte: SIAB, 2013.

Dados do SIAB apontaram que o número de hospitalizações por desnutrição, em crianças menores de 4 anos de idade caíram de 7 em 2008 para nenhum caso em 2012 (Gráfico 3).

Gráfico 3 - Número de hospitalizações por Pneumonia em crianças menores de 4 anos de idade. Ipubi-PE, 2008-2012.

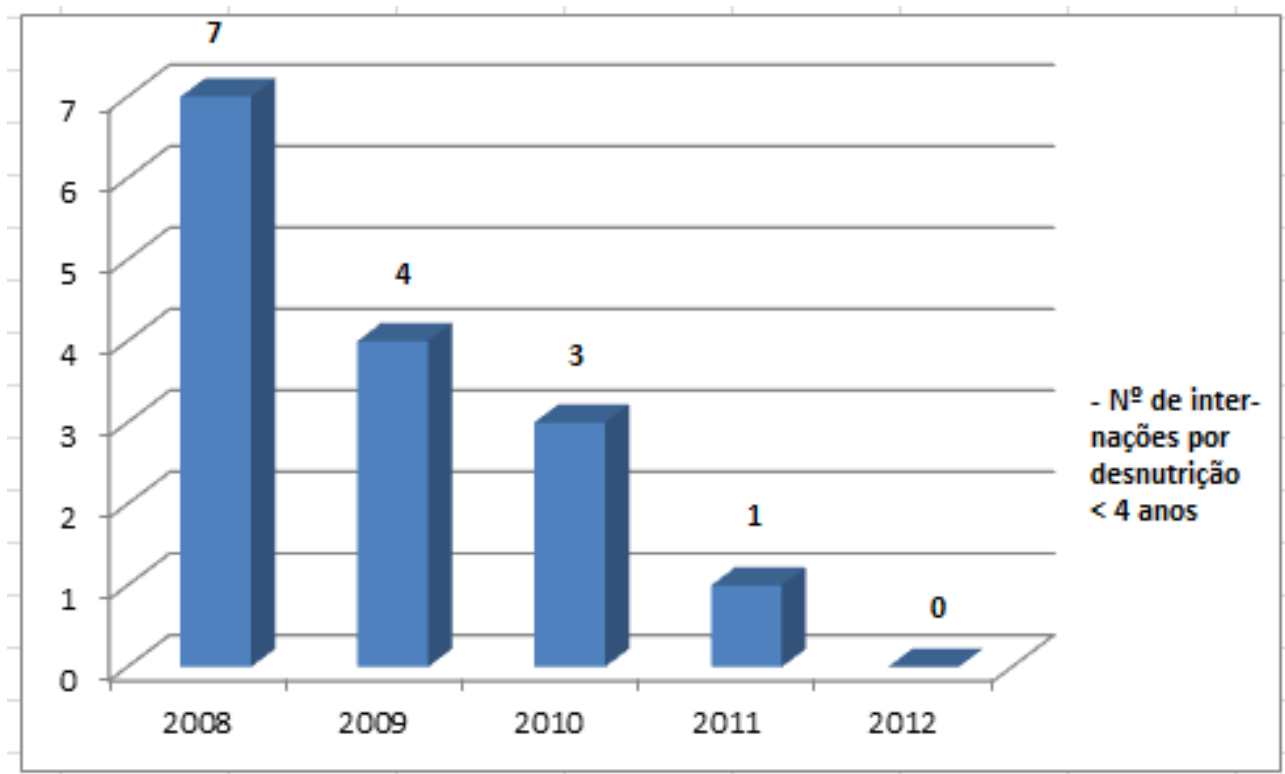

Fonte: SIAB, 2013. 


\section{Conclusão}

A implantação do Programa trouxe benefícios para as crianças, mães e para o próprio município, que reduziu em $67 \%$ (sessenta e sete por cento) os atendimentos e internamentos em crianças menores de 2 (dois) anos, conseqüentemente reduzindo os custos municipais com a saúde, nesta dimensão. Portanto, este trabalho reafirma que o estímulo ao aleitamento materno exclusivo, é uma fórmula relativamente econômica e segura de promoção da saúde, devendo ser incentivada como prioritária, enquanto política pública.

\section{Referências}

ALVES CRL, GOULART EMA, COLOSIMO EA, GOULART LMHF. Fatores de risco para o desmame entre usuárias de uma unidade básica de saúde de Belo Horizonte, Minas Gerais, Brasil, entre 1980 e 2004. Cad Saúde Pública, n.24. p.1355-67, 2008.

BOCCOLINI CS, CARVALHO ML, OLIVEIRA MIC, LEAL MC, CARVALHO MS. Fatores que interferem no tempo entre o nascimento e a primeira mamada. Cad Saúde Pública. n.24. p. 2681-94, 2008.

BAPTISTA GH, ANDRADE AHHKG, GIOLO SR. Fatores associados à duração do aleitamento materno em crianças de famílias de baixa renda da região sul da cidade de Curitiba, Paraná, Brasil. Cad Saúde Pública. n.25.p.596-604, 2009.

BORGES AL, PHILIPPI ST. Opinião de mulheres de uma unidade de saúde da família sobre a quantidade de leite materno produzido. Rev Latinoam Enferm. n.11. p.287-92, 2003.

CARRASCOZA KC. Avaliação dos aspectos biopsicossociais que interferem na amamentação [Dissertação de Mestrado]. Campinas: Universidade Estadual de Campinas; 2007. 
DAMIÃO JJ. Influência da escolaridade e do trabalho maternos no aleitamento materno exclusivo.

Rev Bras Epidemiol. n.11. p.442-52, 2008.

DEARDEN K, ALTAYE IM, OLIVA MSJ, MORROW AL, BURKHALTER BR. Determinants of optimal breast-feeding in peri-urban Guatemala City, Guatemala. Rev Panam Salud Pública. n.12. p.185-92, 2002.

FALEIROS JJ, KALIL G, CASARIN DP, LAQUE JR. PA, SANTOS IS. Avaliação do impacto de um programa de puericultura na promoção da amamentação exclusiva. Cad Saúde Pública 2005; 21:4829.

FRANÇA GVA, BRUNKEN GS, SILVA SM, ESCUDER MM, VENÂNCIO SI. Determinantes da amamentação no primeiro ano de vida em Cuiabá, Mato Grosso. Rev Saúde Pública. n. 41.p. 711-8, 2007.

INSTITUTO BRASILEIRO DE GEOGRAFIA E ESTATÍSTICA - IBGE. Divisão Territorial do Brasil e Limites Territoriais. Instituto Brasileiro de Geografia e Estatística - IBGE: São Paulo, 2008.

KUMMER SC, GIUGLIANE ERJ, SUSIN LO, FOLLETTO JL, LERMEN NR, WU VYJ, et al. Evolução do padrão de aleitamento materno. Rev Saúde Pública. N. 34. P.143-8, 2000.

MASCARENHAS MLW, ALBERNAZ EP, SILVA MB, SILVEIRA RB. Prevalence of exclusive breastfeeding and its determiners in the first 3 months of life in the South of Brazil. J Pediatr (Rio J.). n.82. p.289-94, 2006.

NARCHI NZ, FERNANDES RAQ, GOMES MMF. Análise da efetividade de um programa de incentivo ao aleitamento materno exclusivo em comunidade carente na Cidade de São Paulo. Rev Bras Saúde Matern Infant. n.5:p. 87-92, 2005.

OLIVEIRA MIC, CAMACHO LAB, TEDSTONE AE. A method for the evaluation of primary health care units' practice in the promotion, protection, and support of breastfeeding: results from the State of Rio de Janeiro, Brazil. J Hum Lact. n.19. p.365, 2003. 
PASSOS MC, LAMOUNIER JA, SILVA CAM, FREITAS SSN, BAUDSON MFR. Práticas da amamentação no Município de Ouro Preto, MG, Brasil. Rev Saúde Pública. n.34 .p.617-22, 2000.

RAMOS CV, ALMEIDA JAG, ALBERTO NSMC, TELES JBM, SALDIVA SRDM. Diagnóstico da situação do aleitamento materno no Estado do Piauí, Brasil. Cad Saúde Pública. N. 24. P.1753-62, 2008.

REA MF, VENÂNCIO SI, BATISTA LE, SANTOS RG, GREINER T. Possibilidades e limitações da amamentação entre mulheres trabalhadoras formais. Rev Saúde Pública. n.31. p.149-56, 1997.

SECRETARIA DE ATENÇÃO À SAÚDE, MINISTÉRIO DA SAÚDE. II Pesquisa de prevalência de aleitamento materno nas capitais brasileiras e Distrito Federal. Brasília: Ministério da Saúde; 2009.

SCHWARTZ K, D'ARCY HJ, GILLESPIE B, BOBO J, LONGEWAY M, FOXMAN B. Factors associated with weaning in the first 3 months postpartum. J Fam Pract. n.51.p.439-44, 2002.

VANNUCHI MTO, MONTEIRO CA, RÉA MF, ANDRADE SM, MATSUO T. Iniciativa Hospital Amigo da Criança e aleitamento materno em unidade de neonatologia. Rev Saúde Pública. n.38.p.422-8, 2004.

VIANNA RPT, REA MF, VENÂNCIO SI, ESCUDER MM. A prática de amamentar entre mulheres que exercem trabalho remunerado na Paraíba, Brasil: um estudo transversal. Cad Saúde Pública. n.23.p.2403-9, 2007.

Como citar este artigo (Formato ISO):

MACEDO, C.C; ABREU, L.C.; HENRIQUE, A.M.; CRUZ, M.F.L.; OLIVEIRA, G.F.; CRUZ NETO, J.S.; CRUZ, L.L; MARTINS, M.E.; CARVALHO, S.M.F.. Aleitamento Materno Exclusivo na Região do Araripe, Brasil. Id on Line Revista de Psicologia, Novembro de 2013, vol.1, n.21, p. 35-44. ISSN 1981-1189. 